\title{
Microstrip Patch Antenna Array with Cosecant- Squared Radiation Pattern Profile
}

\author{
K. Kaboutari ${ }^{1, *}$, A. Zabihi ${ }^{2}$, B. Virdee ${ }^{3}$ and M. Pilevari ${ }^{4}$ \\ ${ }^{1}$ Department of Electrical and Electronics Engineering, Middle East Technical \\ University, Ankara, Turkey, (Email: k.kaboutari@hotmail.com) \\ ${ }^{2}$ Department Electrical Engineering, Islamic Azad University, Urmia Branch, Urmia, Iran \\ ${ }^{3}$ Center for Communications Technology, School of Computing \& Digital Media, London Metropolitan University, \\ London N7 8DB, UK \\ ${ }^{4}$ Department of Electrical Engineering, Islamic Azad University, Tehran Jonoob Branch, Tehran, Iran
}

\begin{abstract}
In this paper the radiation pattern on either side of the main beam, which is created by a standard microstrip patch antenna, is configured to correspond to a cosecant-squared curve. The $8 \times 2$ antenna array comprises eight pairs of radiating elements that are arranged in a symmetrical structure and excited through a single common feedline. Interaction of the fields generated by each pair of elements contribute towards the overall radiation characteristics. The proposed array is shown to exhibit an impedance bandwidth of $1.93 \mathrm{GHz}$ from 9.97 to $11.90 \mathrm{GHz}$ for $\mathrm{S}_{11} \leq-10 \mathrm{~dB}$ with a peak gain of $14.95 \mathrm{dBi}$. The antenna's radiation pattern follows a cosecant-squared curve over an angular range of $\pm 60.91^{\circ}$. The compact antenna array has dimensions of $106 \times 34 \times 0.813$ $\mathrm{mm}^{3}$. These characteristics qualify the antenna for radar applications at the X-band frequency.
\end{abstract}

Keywords: Cosecant-squared shaped radiation pattern; Antenna array; X-band applications; Series feedline.

\section{Introduction}

Antennas with a cosecant squared pattern achieve a more uniform signal strength at the input of the receiver as a target moves with a constant height within the beam. In the practice a cosecant squared pattern can be achieved by a deformation of a parabolic reflector [1]. Over the last decade, various techniques have been employed to create cosecant-squared shaped radiation patterns [210], which include utilizing reflector surface [2] and applying multi-feed technique in reflector antennas along with Invasive Weed Optimization (IWO) algorithm [3]. A mechanically leakywave antenna in [4] is used to generate a cosecant-squared radiation pattern. Here the antenna is a superstrate configuration made with a trimmed dielectric layer over a ground plane and fed by a single open-ended horn. Antenna designers have also used array configuration to realize the cosecant-squared shaped radiation pattern [5, 6]. In [5], a corner reflector antenna array is used to create a cosecant-squared shaped radiation pattern in the E-plane. Radiating elements of this antenna, which are made up of four symmetrically arranged pentagonal dipoles, are located parallel to the vertex of a $60^{\circ}$ corner reflector. This antenna has the impedance bandwidth of $1.4 \mathrm{GHz}$ from 8.6 to $12 \mathrm{GHz}$, and $15 \mathrm{dBi}$ peak gain at $10 \mathrm{GHz}$. Planar antenna array structures in [6] use substrate integrated waveguide (SIW), unequal power dividers, and phase-shifters to generate a cosecant- 
squared shaped radiation pattern at $42.6 \mathrm{GHz}$. Here twelve slotted antennas are combined to realize a cosecant-squared shaped pattern.

Nowadays, the use of monopole patch antennas in array structures has become common because of simplicity in design, small form-factor, good radiation characteristics and low-cost fabrication using conventional MIC technology. Various feed techniques and geometries of microstrip antennas have been investigated [7-11]. Patch monopole antennas, however, have constraints in terms of gain and bandwidth. Arranging the patch in an array configuration is a common technique to enhance the antenna's gain [12]. In [13], the gain of a $4 \times 2$ element aperturecoupled antenna array is determined by the mutual coupling between the elements. In this case the gain and impedance bandwidth are $15.7 \mathrm{dBi}$ and $300 \mathrm{MHz}$, respectively. The series-fed microstrip antenna array in [14] has a gain of $6 \mathrm{dBi}$ and impedance bandwidth of $750 \mathrm{MHz}$. In [15], the gain and bandwidth of a multi-layer $2 \times 2$ square patch antenna are improved by implementing a perforated superstrate. The antenna bandwidth is improved by drilling holes in the superstrate layer. The gain and impedance bandwidth of this antenna array are $16 \mathrm{dBi}$ and $4.2 \mathrm{GHz}$, respectively. Although in the prior designs, the gain performance in patch antenna arrays is improved, however, the impedance bandwidth is a limiting factor except in [15]. However, the complexity of a multi-layer design in [15] and fabrication cost are constraining factors. Moreover, different types of the array antennas with various feed networks have been explored recently including a sequentially rotated feed network using branch-line couplers [16] and directional couplers [17] for C-band applications.

In this paper cosecant-squared shaped radiation pattern is realized using a planar antenna array structure. This linearly polarized antenna has a high-gain and broadband performance over its operating frequency range. The symmetrical antenna array structure comprises eight pairs of a square patch and excited with a common microstrip feedline. The number of array patches in the array and the gap between them is shown to determine the antenna's gain and radiation characteristics. Measurements confirm the antenna array has an impedance bandwidth between 9.97 to $11.90 \mathrm{GHz}$ for $\mathrm{S}_{11} \leq-10 \mathrm{~dB}$, and a maximum gain of $14.95 \mathrm{dBi}$.

\section{Single-Element Antenna}

The proposed single-element patch antenna configuration, shown in Fig. 1, consists of a squared patch with two slits cutout in the lower portion of the patch to extend the feed point connection to the patch in order to enhance the matching. In addition, the $50 \Omega$ microstrip feedline is stepped to further improve the impedance match. The other side of the dielectric substrate is a ground plane. The antenna is designed on low-loss Rogers RO4003 substrate with $\varepsilon_{\mathrm{r}}=3.55$ and $\tan \delta=0.0027$. Length and width of the slits and the size of stepped feedline have a critical effect in the antenna's impedance bandwidth. The antenna exhibits a unidirectional radiation pattern along the Z-axis. 


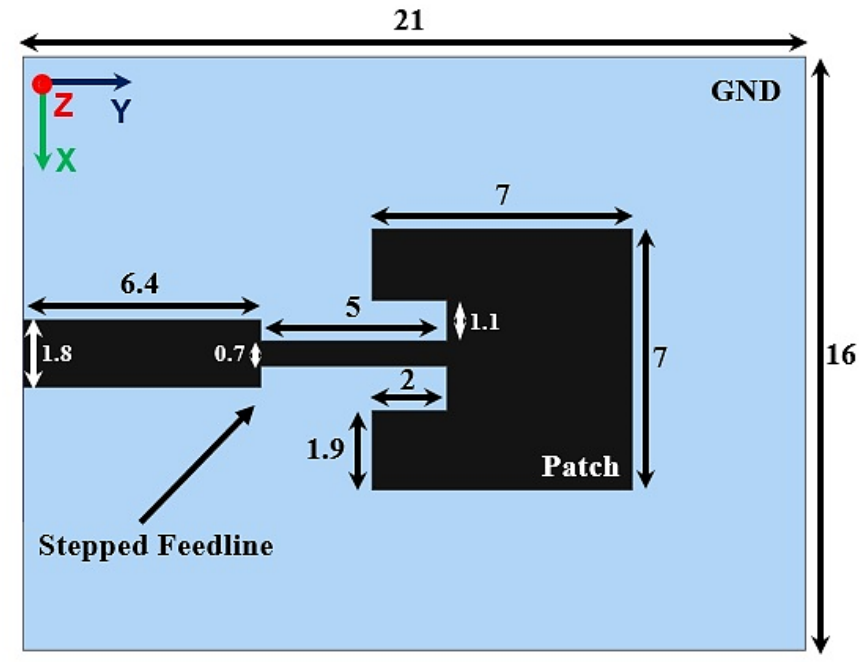

Fig. 1. The proposed single-element patch antenna's structure (dimensions given are in $\mathrm{mm}$ ).

Fig. 2 shows the simulated results of the single-element antenna. All simulations were done using ANSYS HFSS v13. The single-element antenna has an impedance bandwidth of $280 \mathrm{MHz}$ from 10.95 to $11.23 \mathrm{GHz}$, and the corresponding pain varies from 6.4 to $6.9 \mathrm{dBi}$ over the frequency band. A full ground-plane is necessary to achieve a unidirectional radiation pattern. However, there are numerous techniques available to widen the impedance bandwidth of the patch antennas. These techniques can however adversely affect the radiation pattern of the antenna and/or are complicated to implement in practice. Bandwidth enhancing techniques include ground-plane truncation [18] and loading the antenna with resonators [19].

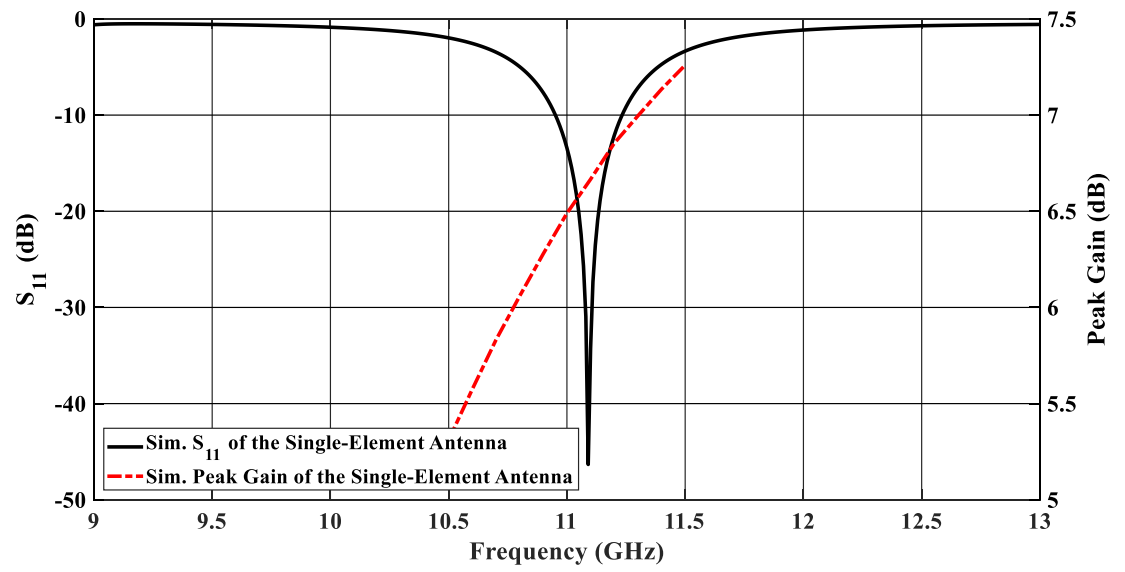

Fig. 2. The simulated $S_{11}$ and the peak gain response of the single-element antenna.

Fig. 3 shows the simulated radiation patterns of the single-element antenna in the XZ- and YZplanes at $11.09 \mathrm{GHz}$. It confirms that the antenna has a stable and unidirectional radiation pattern. The radiation pattern in the YZ-plane is slightly tilted and some radiation occurs below groundplane. This is because an infinite PEC ground-plane was not used in the simulation to be more 
realistic. The consequence of this is excitation of surface waves at the edge of the substrate as well as diffraction of the radiated field.

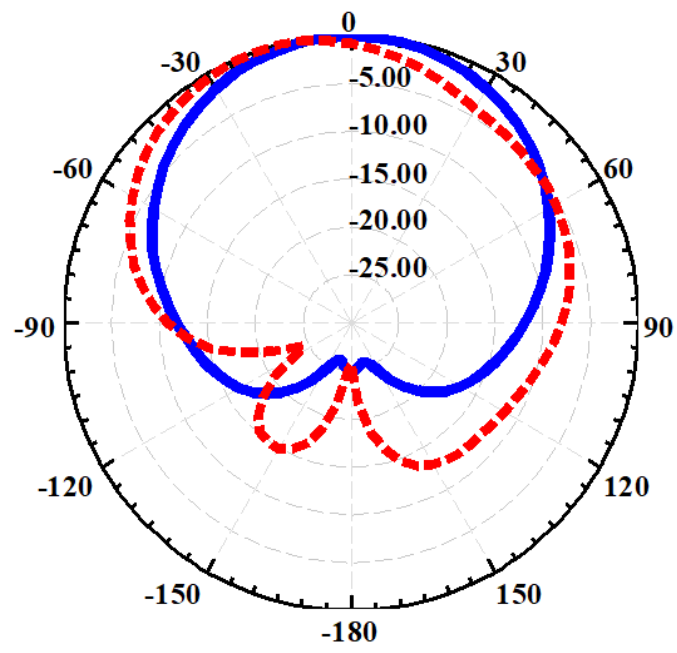

Fig. 3. Simulated radiation patterns of the single-element antenna at $11.09 \mathrm{GHz}$ (solid line at XZ-plane, and dash line at YZ-plane).

\section{Antenna Array Configuration}

The proposed antenna structure is very general and lends itself to compressive-sensing-based diagnostics applications [20]. The single-element patch antenna in Fig. 1 was used to realize the antenna array, which is shown in Fig. 4. This symmetrical configuration along the Y-axis is shown to exhibit a cosecant-squared shaped radiation pattern from the antenna. The array comprises 16elements in $8 \times 2$ configuration, and the radiation elements are excited with a common feedline. Power from the feedline is divided equally between the radiation elements. A parametric study was conducted to determine the gap between the radiation elements to realize the required cosecant-squared radiation pattern. The optimized dimensions of the array structure are annotated in Fig. 4. These values are given in millimeters.

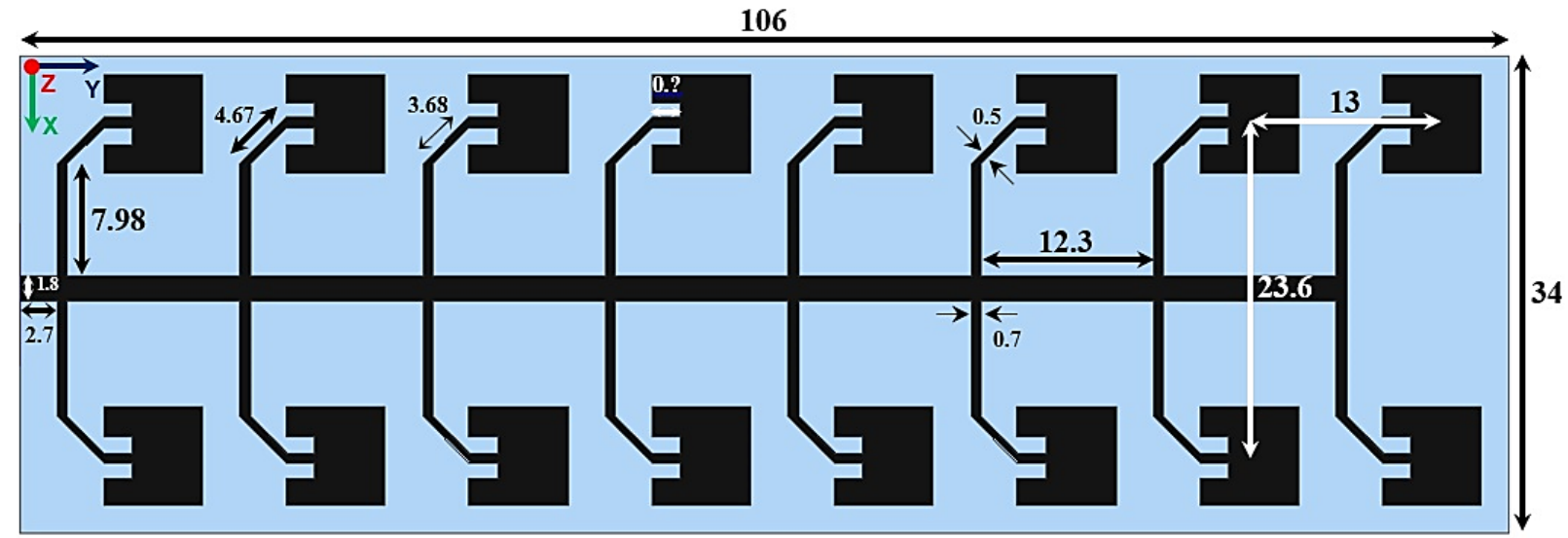

Fig. 4. Structure of the proposed antenna array with 16-elements (dimensions given are in mm). 
In the proposed array configuration higher transmission power will be delivered to the lower patches than to the upper patches. This unequal power distribution along the patches will have an impact on the array's radiation pattern. Parametric study was carried out to determine the optimum performance from the proposed antenna array. The return-loss and normalized radiation pattern of the antenna array are plotted in Fig. 5 \& 6 for 4, 8, 12 and 16-element arrays. It is evident from theses plots the greater the number of elements the better the impedance bandwidth. In this study it was found that 16-element array provided the best performance. Indeed, the impedance bandwidth of the antenna array increase from $520 \mathrm{MHz}$ for 4-element to $1840 \mathrm{MHz}$ for 16-element. It was discovered that by increasing the antenna array elements greater than 16 -element had negligible effect on the impedance bandwidth. The empirical results also suggest that surface waves propagating over the antenna cause mutual coupling effects between the radiating elements that perturb the intrinsic resonant frequency of the elements that contributes in the resulting bandwidth of the array antenna. Surface waves contributing to this are TM and TE modes of the substrate. This has the effect of suppressing grating lobe at $11.90 \mathrm{GHz}$.

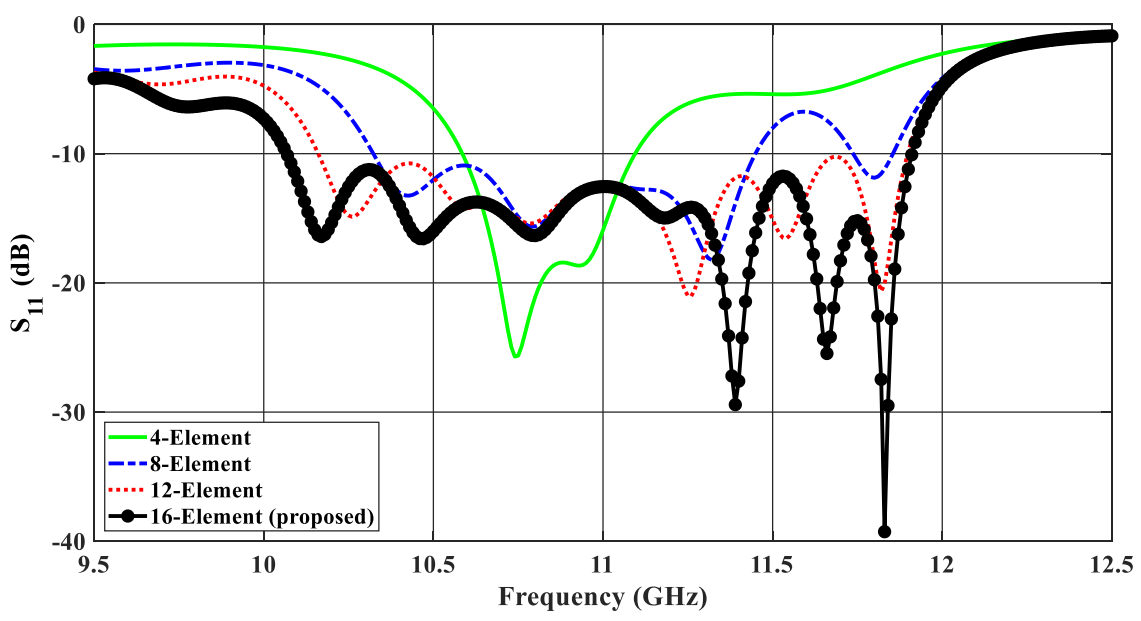

Fig. 5. Return-loss response of the antenna array as a function of array elements $(4,8,12 \& 16$-elements).




Fig. 6. Normalized radiation pattern of the antenna array as a function of array elements $(4,8,12 \& 16$-elements) in the YZ-plane at $11.25 \mathrm{GHz}$.

Fig. 7 shows how the radiation pattern of the array was influenced by the number of array elements. By increasing the number of elements in the antenna array, the radiation pattern becomes narrower and follows a cosecant-squared shaped function. The simulated radiation pattern of the proposed 16-element antenna array in the XZ- and YZ-planes at $11.25 \mathrm{GHz}$ are shown in Fig. 7.



Fig. 7. Simulated radiation pattern of the antenna array at $11.25 \mathrm{GHz}$ (solid line in the XZ-plane, and dash line in the YZ-plane).

Fig. 8 shows the simulated far-field radiation patterns of the proposed antenna and the co- and cross-polarization in the H-plane (XZ-plane) and E-plane (YZ-plane) at $10.95 \mathrm{GHz}$. The co and cross-pol radiation patterns for both single and sixteen-element array indicate that the main radiation is at least $20 \mathrm{~dB}$ greater than the unwanted radiation pattern in the broadside direction (Theta and Phi are equal to zero degrees). The results show the single and array antennas have a predominantly unidirectional radiation pattern.

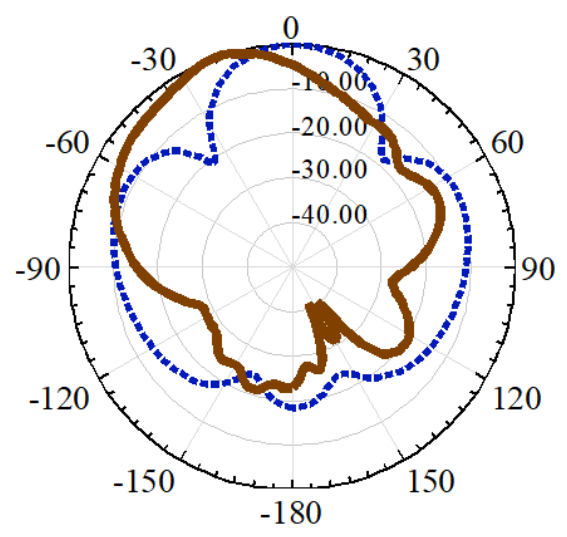

Radiation pattern of the sixteen-element array antenna at $10.95 \mathrm{GHz}$ (solid-line in the XZ-plane, and dash-line in the YZ-plane). 

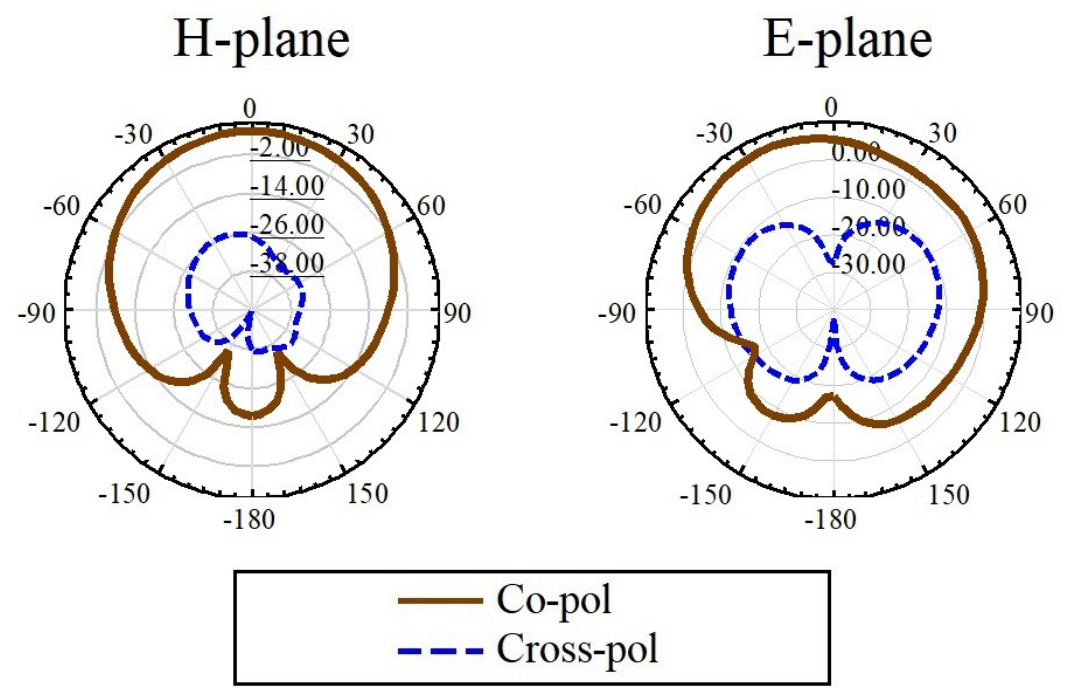

Co and Cross-pol radiation pattern of the single-element array antenna at $10.95 \mathrm{GHz}$.
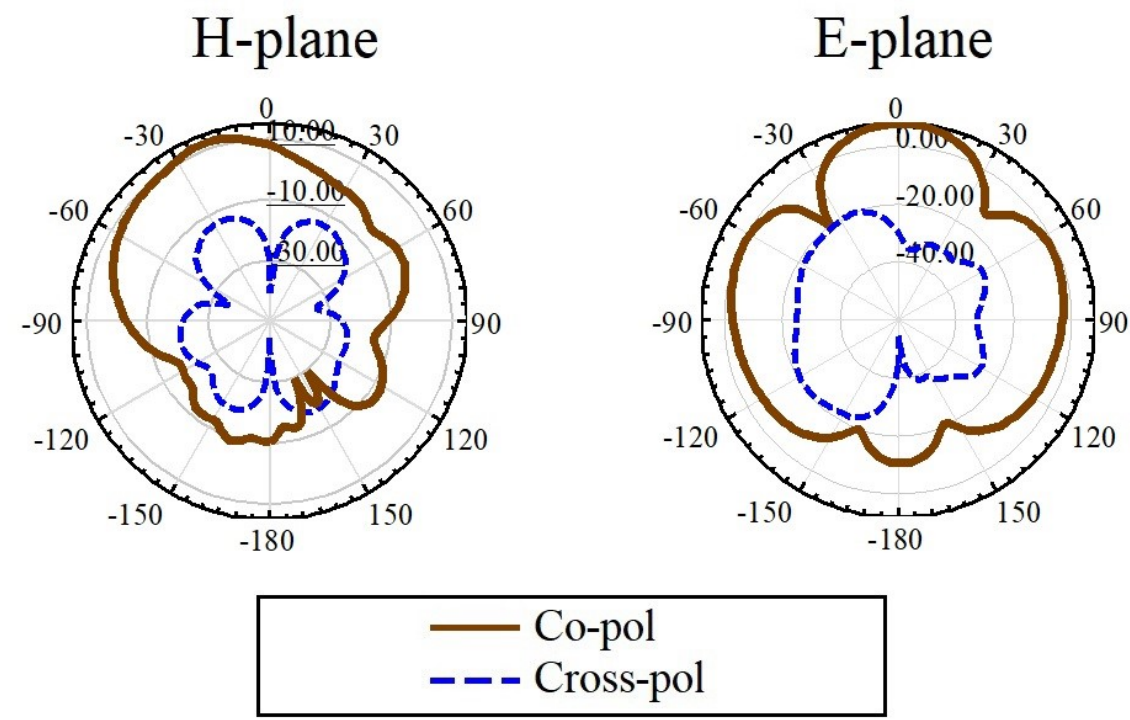

Co and Cross-pol radiation pattern of the sixteen-element array antenna at $10.95 \mathrm{GHz}$.

Fig. 8. Simulated far-field radiation patterns and co- and cross-polarization in the H-plane (XZ-plane) and Eplane (YZ-plane) at $10.95 \mathrm{GHz}$.

A prototype of the finalized antenna array was fabricated and tested in an anechoic chamber. 8722ES Network Analyzer was used to measure its return-loss performance. Comparison between the simulated and measured results of the antenna array shown in Fig. 9 and 10. The measured impedance bandwidth of the antenna array is $1.93 \mathrm{GHz}$ from 9.97 to $11.90 \mathrm{GHz}$. The normalized radiation pattern of the simulated and measured antenna array shows a close correspondence with a standard cosecant-squared curve, as shown in Fig. 10. 


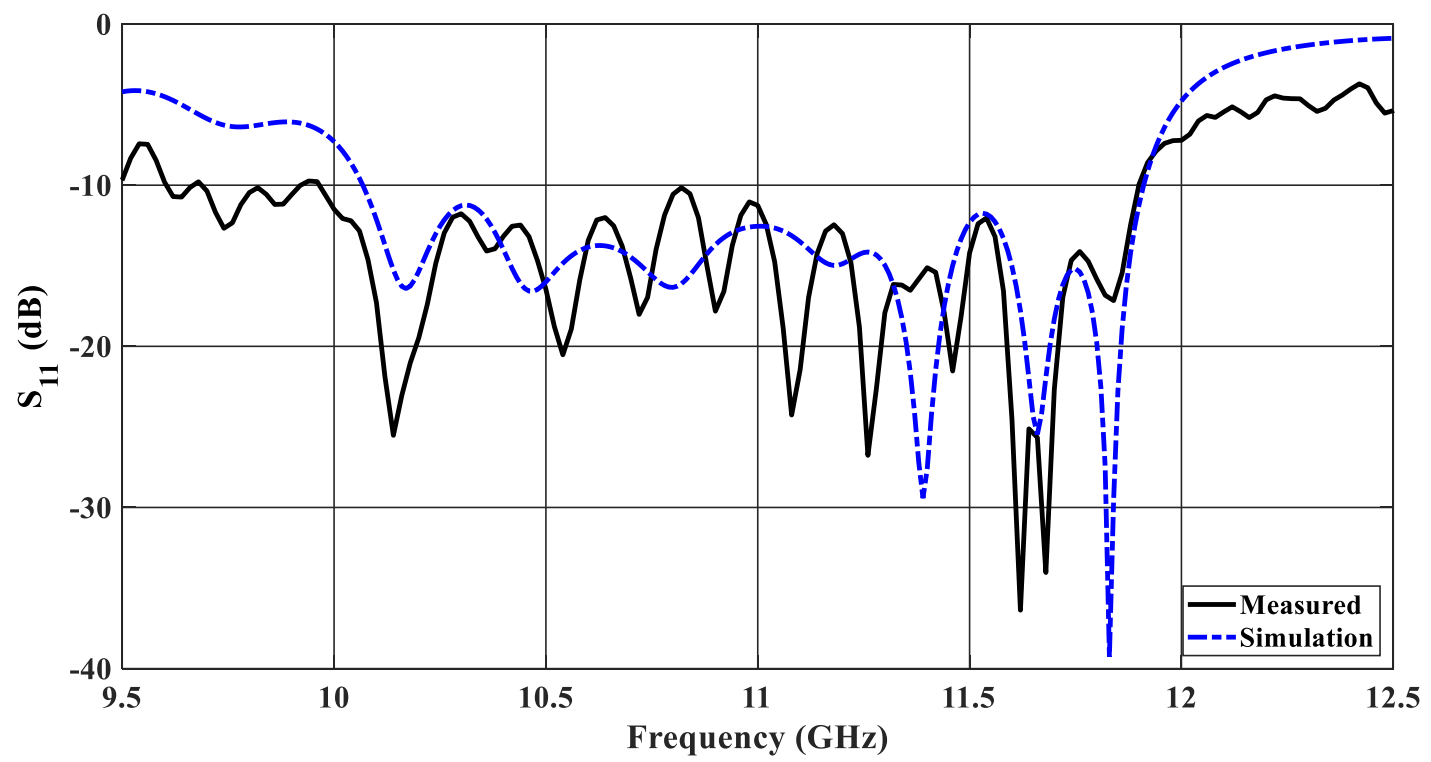

Fig. 9. Measured and simulated return-loss response of the antenna array.

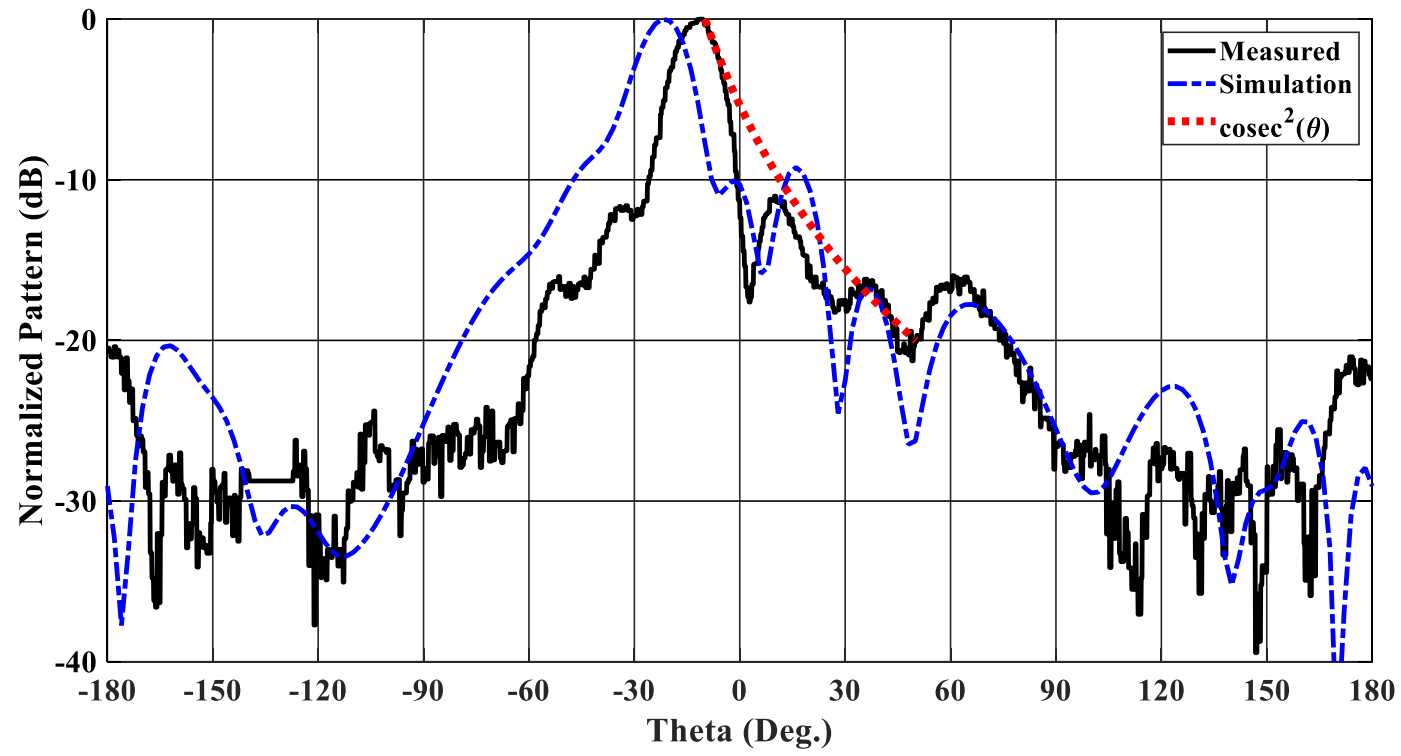

Fig. 10. Measured and simulated normalized radiation patterns of the antenna array in the YZ-plane at $11.25 \mathrm{GHz}$.

The antenna's radiation pattern is in the YZ-plane at $11.25 \mathrm{GHz}$. It follows a cosecant-squared curve over an angular range of $\pm 60.91^{\circ}$ with the main beam centered at $\theta=-11.55^{\circ}$. The sidebands are attenuated by more than $10 \mathrm{~dB}$ either side of the main beam. The 3D radiation pattern of the antenna array at $11.25 \mathrm{GHz}$ is shown in in Fig. 11. 


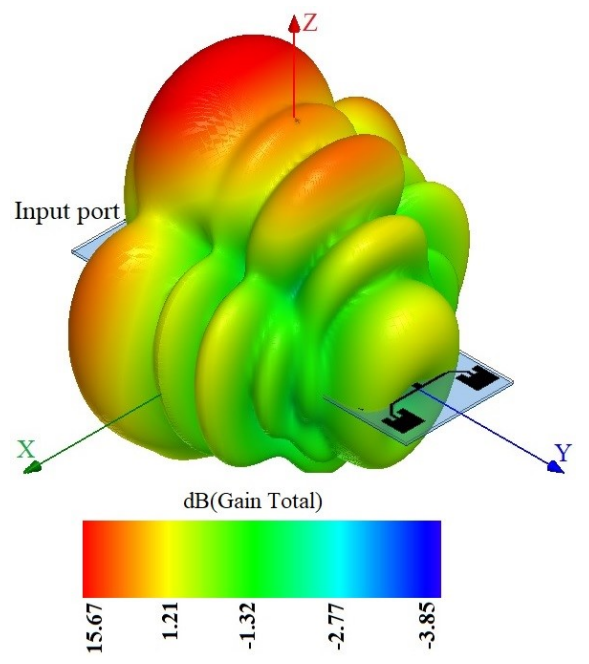

(a)

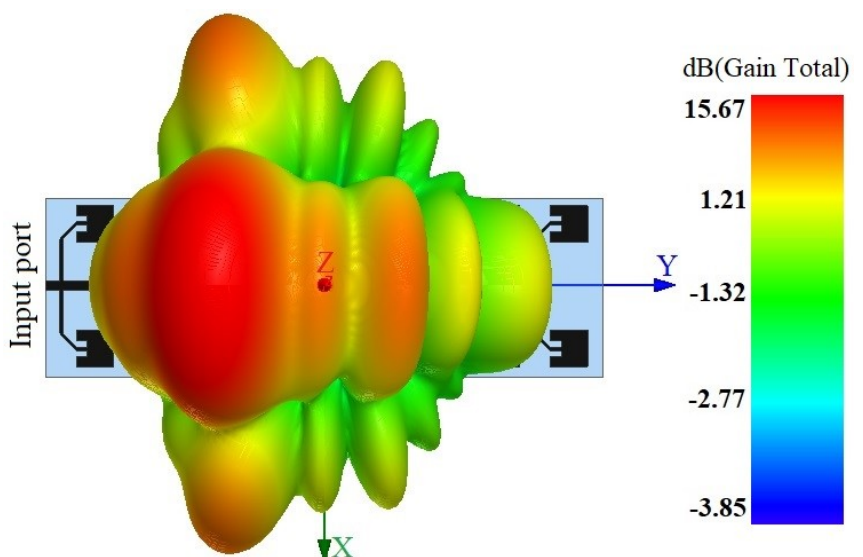

(b)

Fig. 11. Simulated radiation pattern of the antenna array at $11.25 \mathrm{GHz}$, (a) Simulated 3D radiation pattern (isometric view), and (b) Simulated radiation pattern in the XY-plane.

It is evident from Fig. 11 the elements nearer the input of the feedline are more energized and therefore have a radiate greater power. It was essential to ensure the gap between adjacent pairs of elements was adjusted correctly for proper phase shifting to realize the required radiation pattern that followed the cosecant-squared curve. Fig. 12 shows the simulated and measured peak gain of the realized antenna array in comparison with the single-element antenna gain.

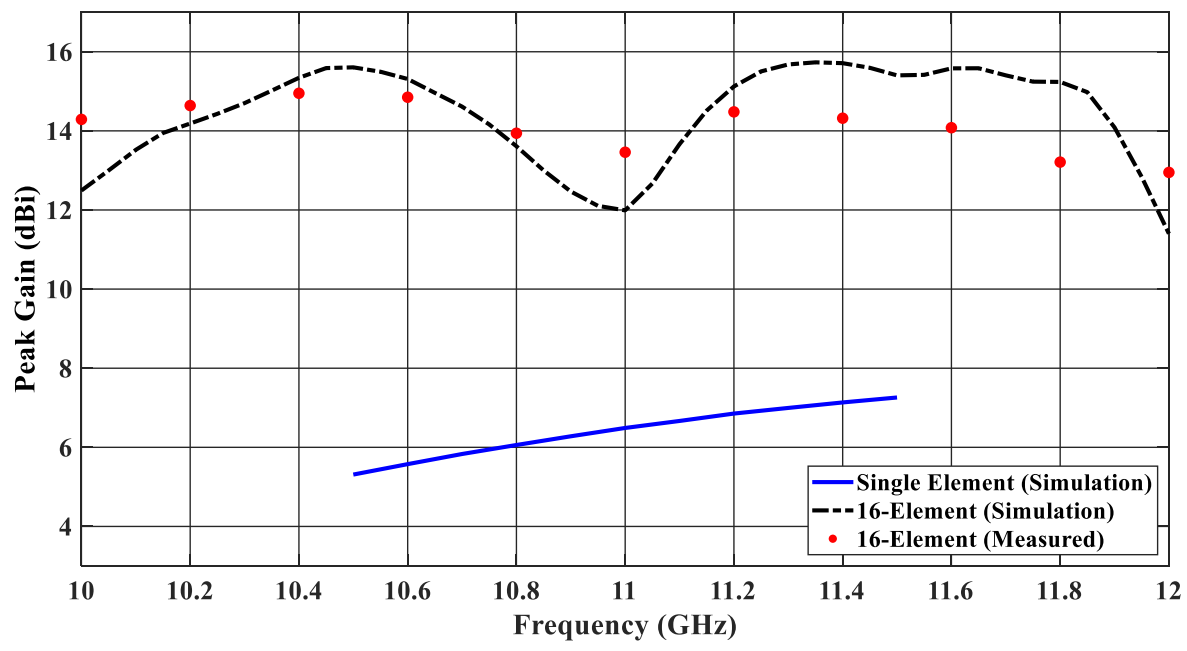

Fig. 12. Peak gain performance of the antenna.

The average peak radiation gain increases from $6.62 \mathrm{dBi}$ for the single-element antenna to approximately $14.22 \mathrm{dBi}$ for the 16-element antenna array. Moreover, the antenna array has maximum measured gain of $14.95 \mathrm{dBi}$ at $10.40 \mathrm{GHz}$. The measurement setup used is shown in Fig. 13. A standard technique was used to measure the antenna's radiation pattern in the anechoic chamber. 


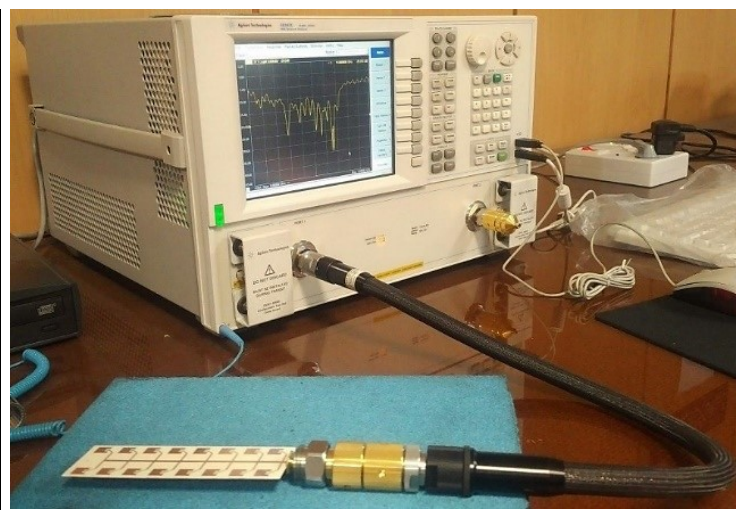

(a)

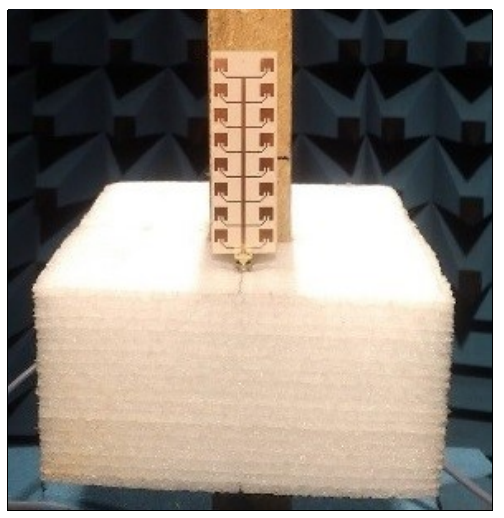

(b)

Fig. 13. Measurement setup for the fabricated antenna array, (a) Antenna array connected to the network analyzer, and (b) Antenna array in the anechoic chamber.

\section{Conclusion}

A series fed $8 \times 2$ antenna array, which is constructed from standard square patches, is shown to exhibit a radiation pattern with a sidelobe profile that follows a cosecant-squared curve. Effect on the array's impedance match and radiation patterns as a function of number of elements constituting the array was investigated. Average gain of the linearly polarized antenna array and its impedance bandwidth are $14.22 \mathrm{dBi}$ and $1.93 \mathrm{GHz}$, respectively. The antenna array is compact with dimensions of $106 \times 34 \times 0.813 \mathrm{~mm}^{3}$ when fabricated on Rogers RO4003 dielectric substrate. There is close correspondence between the measured and simulated results.

\section{Acknowledgement}

The authors would like to thank the Northwest Antenna and Microwave Research Laboratory (NAMRL) at Urmia University for technical supporting. We would like to express our deep thanks to our dear friend Majid Shokri, who blessed us with his priceless and unforgettable supports throughout all our studies. We would like to extend our gratitude to Mahsa Karimabadi for her assistance in the technical writing of the paper.

\section{References}

[1] M. Skolnik, Radar Handbook, New York, NY, USA, McGraw-Hill, 1970.

[2] S. Karimkashi, A. R. Mallahzadeh, J. Rashed-Mohassel, “A New Shaped Reflector Antenna for Wide Beam Radiation Patterns,” Proc. Int. Symp. MAPE, Hangzhou, China, 2007, pp. 535-538.

[3] A. Foudazi, A. R. Mallahzadeh, M. M. Samadi Taheri, "Pattern Synthesis for Multi-Feed Reflector Antenna Using IWO Algorithm,” Proc. 6th Eur. Conf. Antennas Propag., Prague, Czech Republic, 2012, pp. 1-5.

[4] F. Scattone, M. Ettorre, B. Eddo, R. Sauleau and N. J. G. Fonseca, "Truncated Leaky-Wave Antenna with Cosecant-Squared Radiation Pattern," IEEE Antennas and Wireless Propagation Letters, vol. 17, no. 5, pp. 841844, May 2018.

[5] M. Milijić, A. D. Nešić and B. Milovanović, "Design, Realization, and Measurements of a Corner Reflector Printed Antenna Array with Cosecant Squared-Shaped Beam Pattern," IEEE Antennas and Wireless Propagation Letters, vol. 15, pp. 421-424, 2016. 
[6] Z. Hao and M. He, "Developing Millimeter-Wave Planar Antenna with a Cosecant Squared Pattern," IEEE Transactions on Antennas and Propagation, vol. 65, no. 10, pp. 5565-5570, Oct. 2017.

[7] M. Ojaroudi, C. Ghobadi, and J. Nourinia, "Small square Monopole Antenna with Inverted T-Shaped Notch in the Ground Plane for UWB Application," IEEE Antennas Wireless Propag. Lett., vol. 8, July 2009, pp. 728-731.

[8] M. T. I. Huque, M. K. Hosain, M. S. Islam, and M. Al-Chowdhury, "Design and Performance Analysis of Microstrip Array Antennas with Optimum Parameters for X-band Applications," Int. J. of Advanced Computer Science and Appl., vol.3, no. 6, 2015, pp. 416-423.

[9] G. Kaur, J. Singh, "H and T-Slotted Rectangular Microstrip Patch Antennas for Wireless Communication," Int. J. of Computer Applications, vol. 118, no. 8, 2015.

[10] Y. Coulibaly, T. A. Denidni and H. Boutayeb, "Broadband Microstrip-Fed Dielectric Resonator Antenna for XBand Applications," IEEE Antennas and Wireless Propagation Letters, vol. 7, 2008, pp. 341-345.

[11] J. C. S. Chieh and A. V. Pham, "A Bidirectional Microstrip X-Band Antenna Array on Liquid Crystal Polymer for Beamforming Applications," IEEE Trans. on Antennas and Prop., vol. 61, no. 6, June 2013, pp. 3364-3368.

[12] I. Mohamed and A. R. Sebak, "High-Gain Series-Fed Aperture-Coupled Microstrip Antenna Array," Microwave and Optical Tech. Letters, vol. 57, no. 1, 2015, pp. 91-94.

[13] X. Lin, M. Luo, Y. Yang, and K. Huang, "High-Gain Aperture-Coupled Microstrip Array Antenna," IEEE International Conference on Microwave and Millimeter Wave Technology 2012 (ICMMT), vol.5, 2012, pp. 14.

[14] B Zivanovic, T. M. Weller and C. Costas, "Series-Fed Microstrip Antenna Arrays and Their Application to Omnidirectional Antennas," IEEE Trans. Antennas Propag., 2012, pp.4954-4959.

[15] M. Asaadi, and A. Sebak, "Gain and Bandwidth Enhancement of $2 \times 2$ Square Dense Dielectric Patch Antenna Array Using a Holey Superstrate," IEEE Antennas and Wireless Propag. Lett., vol. 16, 2017, pp.1808-1811.

[16] Siahcheshm A, Nourinia J, Ghobadi Ch, Karamirad M, and Mohammadi B., "A Broadband Circularly Polarized Cavity-Backed Archimedean Spiral Array Antenna for C-Band Applications," AEU - International Journal of Electronics and Communications, vol. 81, 2017, pp.218-226.

[17] A. Siahcheshm, J. Nourinia, Ch. Ghobadi, "Circularly Polarized Antenna Array with a New Sequential Phase Feed Network Utilizing Directional Coupler," AEU - International Journal of Electronics and Communications, vol. 93, 2018, pp.75-82.

[18] T. Ali, B. K. Subhash, S. Pathan, R. C. Biradar, "A Compact Decagonal-Shaped UWB Monopole Planar Antenna With Truncated Ground Plane," Microwave and Optical Tech. Letters, Oct. 2018, pp.2937-2944.

[19] F. Alizadeh, C. Ghobadi, J. Nourinia, R. Zayer, "Bandwidth Enhancement of Patch Antennas Loaded with Complementary Split-Ring Resonators," 7th Int. Symposium on Telecommunications (IST'2014), 2014, pp. 224229.

[20] A. F. Morabito, R. Palmeri, and. T. Isernia, "A Compressive-Sensing-Inspired Procedure for Array Antenna Diagnostics by a Small Number of Phaseless Measurements," IEEE Transactions on Antennas and Propagation, vol. 64, n. 7, pp. 3260-3265, 2016. 